\title{
Correction to: Contributing factors to severe complications after liver resection: an aggregate root cause analysis in 105 consecutive patients
}

Kholoud Houssaini ${ }^{1 *}$, Oumayma Lahnaoui ${ }^{1}$, Amine Souadka', Mohammed Anass Majbar ${ }^{1}$, Abdelilah Ghannam², Brahim El Ahmadi², Zakaria Belkhadir ${ }^{2}$, Laila Amrani ${ }^{1}$, Raouf Mohsine ${ }^{1}$ and Amine Benkabbou ${ }^{1}$

Correction to: Patient Saf Surg 14, 36 (2020)

https://doi.org/10.1186/s13037-020-00261-7

Following publication of the original article [1], the author would like to make the below two corrections:

- Change Table 3 (with shaded color).

- Change the below author names:

- Mohammed Anass Majbar

- Laila Amrani

- Abdelilah Ghannam

The original article has been updated as well.

\author{
Author details \\ 'Surgical Oncology Department, National Institute of Oncology, \\ MohammedV University in Rabat, Rabat, Morocco. ${ }^{2}$ Intensive Care \\ Department, Nationallnstitute of Oncology, Mohammed V University in \\ Rabat, Rabat, Morocco.
}

Published online: 12 November 2020

\section{Reference}

1. Houssaini K, Lahnaoui O, Souadka A, et al. Contributing factors to severe complications after liver resection: an aggregate root cause analysis in 105 consecutive patients. Patient Saf Surg. 2020;14:36 https://doi.org/10.1186/ s13037-020-00261-7.

The original article can be found online at https://doi.org/10.1186/s13037020-00261-7.

* Correspondence: houssainikholoud@gmail.com

'Surgical Oncology Department, National Institute of Oncology, MohammedV University in Rabat, Rabat, Morocco

Full list of author information is available at the end of the article

(c) The Author(s). 2020 Open Access This article is licensed under a Creative Commons Attribution 4.0 International License, which permits use, sharing, adaptation, distribution and reproduction in any medium or format, as long as you give appropriate credit to the original author(s) and the source, provide a link to the Creative Commons licence, and indicate if changes were made. The images or other third party material in this article are included in the article's Creative Commons licence, unless indicated otherwise in a credit line to the material. If material is not included in the article's Creative Commons licence and your intended use is not permitted by statutory regulation or exceeds the permitted use, you will need to obtain permission directly from the copyright holder. To view a copy of this licence, visit http://creativecommons.org/licenses/by/4.0/. The Creative Commons Public Domain Dedication waiver (http://creativecommons.org/publicdomain/zero/1.0/) applies to the data made available in this article, unless otherwise stated in a credit line to the data. 
Table 3 Combinations of triggered contributory factors across the 15 single cases

\begin{tabular}{|c|c|c|c|c|c|c|c|c|c|c|c|c|c|c|c|c|c|}
\hline \multicolumn{3}{|c|}{ ALARM categories } & \multicolumn{15}{|c|}{ Triggered contributory factors } \\
\hline \begin{tabular}{|l|} 
Category description \\
\end{tabular} & Subcategory description & Factors & Case 1 & \begin{tabular}{|l|} 
Case 2 \\
\end{tabular} & \begin{tabular}{|c|c|c|c|} 
Case $3^{*}$ \\
\end{tabular} & \begin{tabular}{|l|} 
Case 4 \\
\end{tabular} & \begin{tabular}{|l|} 
Case 5 \\
\end{tabular} & \begin{tabular}{|l|l} 
Case 6 \\
\end{tabular} & \begin{tabular}{|c|} 
Case 7 \\
\end{tabular} & Case $8^{*}$ & \begin{tabular}{|l|} 
Case 9 \\
\end{tabular} & \begin{tabular}{|l|} 
Case 10 \\
\end{tabular} & Case 11* & \begin{tabular}{|l|l|} 
Case 12 \\
\end{tabular} & \begin{tabular}{|c|} 
Case 13 \\
\end{tabular} & 3 Case $14^{4} \mathrm{C}$ & Case $15^{\circ}$ \\
\hline \multirow{9}{*}{$\begin{array}{l}\text { I. PATIENT } \\
\text { FACTORS }\end{array}$} & 1.1 Medical history & Q1 & & & & & & & & & & & & & & & \\
\hline & \multirow{3}{*}{1.2 Health status } & Q2 & & & & & & & & & & & & & & & \\
\hline & & Q3 & & & & & & & & & & & & & & & \\
\hline & & Q4 & & & & & & & & & & & & & & & \\
\hline & 1.3 Medications & Q5 & & & & & & & & & & & & & & & \\
\hline & \multirow{2}{*}{ 1.4 Personality, social and familial factors } & Q6 & & & & & & & & & & & & & & & \\
\hline & & Q7 & & & & & & & & & & & & & & & \\
\hline & \multirow{2}{*}{1.5 Conflictual relationships } & Q8 & & & & & & & & & & & & & & & \\
\hline & & Q9 & & & & & & & & & & & & & & & \\
\hline \multirow{8}{*}{ II. TASK FACTORS } & 2.1 Protocols availability and use & Q10 & & & & & & & & & & & & & & & \\
\hline & \multirow{3}{*}{ 2.2 Test results availability and accuracy } & Q11 & & & & & & & & & & & & & & & \\
\hline & & Q12 & & & & & & & & & & & & & & & \\
\hline & & Q13 & & & & & & & & & & & & & & & \\
\hline & 2.3 Tasks design and clarity & Q14 & & & & & & & & & & & & & & & \\
\hline & \multirow{3}{*}{ 2.4 Planning and schedulling } & Q15 & & & & & & & & & & & & & & & \\
\hline & & Q16 & & & & & & & & & & & & & & & \\
\hline & & Q17 & & & & & & & & & & & & & & & \\
\hline \multirow{4}{*}{$\begin{array}{c}\text { III. INDIVIDUAL (STAFF) } \\
\text { FACTORS }\end{array}$} & \multirow{3}{*}{3.1 Competence, technical and non-technical skills } & Q18 & & & & & & & & & & & & & & & \\
\hline & & Q19 & & & & & & & & & & & & & & & \\
\hline & & Q20 & & & & & & & & & & & & & & & \\
\hline & 3.2 Physical and mental health & Q21 & & & & & & & & & & & & & & & \\
\hline \multirow{9}{*}{$\begin{array}{l}\text { IV.TEAM } \\
\text { FACTORS }\end{array}$} & \multirow{2}{*}{4.1 Communication with staff } & Q22 & & & & & & & & & & & & & & & \\
\hline & & Q23 & & & & & & & & & & & & & & & \\
\hline & 4.2 Communication with patient and family & Q24 & & & & & & & & & & & & & & & \\
\hline & 43 Patient's record & Q25 & & & & & & & & & & & & & & & \\
\hline & 4.3 Pallents record & Q26 & & & & & & & & & & & & & & & \\
\hline & 4.4 Crucial information sharing & Q27 & & & & & & & & & & & & & & & \\
\hline & 4.5 Supervision & Q28 & & & & & & & & & & & & & & & \\
\hline & 4.6 Support & Q29 & & & & & & & & & & & & & & & \\
\hline & 4.6 supporn & Q30 & & & & & & & & & & & & & & & \\
\hline & 5.1 Physical environment maintenance and hygiene & Q31 & & & & & & & & & & & & & & & \\
\hline & 5.2 Patient transfer & Q32 & & & & & & & & & & & & & & & \\
\hline & & Q33 & & & & & & & & & & & & & & & \\
\hline & $\begin{array}{l}5.3 \text { Supplies and equipment design, availability and } \\
\text { maintenance }\end{array}$ & Q34 & & & & & & & & & & & & & & & \\
\hline & & Q35 & & & & & & & & & & & & & & & \\
\hline $\begin{array}{l}\text { V. WORK } \\
\text { ENVIRONMENT }\end{array}$ & 5.4 Computized Information system & Q36 & & & & & & & & & & & & & & & \\
\hline FACTORS & 5.5 Staffing levels and skills mix & Q37 & & & & & & & & & & & & & & & \\
\hline & & Q38 & & & & & & & & & & & & & & & \\
\hline & 56 Workload & Q39 & & & & & & & & & & & & & & & \\
\hline & 5.6 Workload & Q40 & & & & & & & & & & & & & & & \\
\hline & 5.7 Procedures timing & Q41 & & & & & & & & & & & & & & & \\
\hline & 5.7 Procedures timing & Q42 & & & & & & & & & & & & & & & \\
\hline & 6.1 Organizational structure & Q43 & & & & & & & & & & & & & & & \\
\hline & 6.2 Human resources & Q44 & & & & & & & & & & & & & & & \\
\hline & 6.3 Policy, standards and goals & Q45 & & & & & & & & & & & & & & & \\
\hline VI. MANAGEMENT AND & 6.4 Subcontracting management & Q46 & & & & & & & & & & & & & & & \\
\hline CONTEXT FACTORS & 6.5 Purchasing policy & Q47 & & & & & & & & & & & & & & & \\
\hline & 6.6 Adverse event declaration and prevention & Q48 & & & & & & & & & & & & & & & \\
\hline & 6.7 Financial resources & Q49 & & & & & & & & & & & & & & & \\
\hline & 6.8. Wider health service environment & Q50 & & & & & & & & & & & & & & & \\
\hline & Triggered contributory factor, $\mathrm{N}(\%)$ & & $16(329$ & $11(22 \%)$ & $20(40 \%)$ & $8(16 \%)$ & $8(16 \%)$ & $12(24 \%)$ & $8(16 \%)$ & $8(16 \%)$ & $8(18 \%)$ & $6(12 \%)$ & $17\left(34^{\circ}\right.$ & $10(20 \%)$ & $4(8 \%)$ & $15(30 \%) 1$ & $11(22 \%)$ \\
\hline & Non applicable, $\mathrm{N}$ & & 2 & 1 & 5 & 1 & 1 & 2 & 0 & 1 & 1 & 1 & 1 & 0 & 0 & 0 & 0 \\
\hline & & & Case 1 & Case 2 & Case $3^{*}$ & \begin{tabular}{|l|} 
Case 4 \\
\end{tabular} & \begin{tabular}{|l|l|} 
Case 5 \\
\end{tabular} & \begin{tabular}{|l|l|} 
Case 6 \\
\end{tabular} & Case 7 & Case $8^{*}$ & \begin{tabular}{|c|} 
Case 9 \\
\end{tabular} & Case 10 & Case 1 & \begin{tabular}{|l|l|} 
Case 12 \\
\end{tabular} & Case 13 & 3 Case $14^{4} \mathrm{C}$ & Case 15" \\
\hline
\end{tabular}

